\title{
Análisis de grupos de trabajo virtuales y su relación con el aprendizaje colaborativo de la matemática en estudiantes universitarios
}

\section{Analysis of Virtual Work Groups and Their Relationship with Collaborative Learning of Mathematics in University Students}

\author{
Alejandro M. Ecos Espino \\ Universidad Nacional de Moquegua, Moquegua, Perú \\ ORCID: https://orcid.org/0000-0002-3452-5388 \\ Zoraida R. Manrique Chávez \\ Universidad Nacional de la Amazonía, Pucallpa, Perú \\ ORCID: https://orcid.org/0000-0002-0899-8747 \\ Joffré Huamán Núñez \\ Universidad Nacional Micaela Bastidas de Apurímac, Abancay, Perú \\ ORCID: https://orcid.org/0000-0001-9315-5228
}

Received 07-31-20 Revised 08-12-20 Accepted 09-28-20 On line 09-30-20

*Correspondence

Email: aecose@unam.edu.pe
Cite as:

Ecos, A., Manrique, Z., \& Huamán, J. (2020). Análisis de grupos de trabajo virtuales y su relación con el aprendizaje colaborativo de la matemática en estudiantes universitarios. Propósitos y Representaciones, 8 (SPE3), e595. Doi: http://dx.doi.org/10.20511/pyr2020.v8nSPE3.595 


\section{Resumen}

El objetivo de la presente investigación fue determinar la relación entre la participación de los estudiantes, sus estrategias de aprendizaje y la estructura de organización colaborativa de los grupos, con su aprendizaje de la matemática tanto a nivel individual como grupal, en una tarea grupal virtual de tipo colaborativa, dentro del contexto de la emergencia sanitaria generada por el COVID19. El diseño de la investigación fue no experimental transversal de tipo descriptivo correlacional. La población de estudio estuvo conformada por 365 estudiantes de la carrera de Ingeniería Ambiental de la Universidad Nacional de Moquegua, con una muestra intencional de 48 estudiantes matriculados en el curso Cálculo I, los cuales formaron 16 grupos de trabajo compuestos por 3 integrantes, quienes trabajaron durante 4 semanas, la resolución de 4 situaciones problemáticas relacionadas a la variación y el cambio de las funciones. La entrega de la información se realizó a través de la plataforma de la universidad y la reunión de grupos se efectuó a través de la herramienta WhatsApp. Los resultados indican que la mejora en el entendimiento, manejo y traducción de distintas representaciones de un objeto matemático así como de la interpretación y manipulación de las expresiones matemáticas tiene que ver con las participaciones de los estudiantes dentro del grupo virtual de trabajo relacionadas con el contenido de la tarea y que los grupos donde se produce una mejora en el aprendizaje de la resolución de problemas son aquello que desarrollan una estructura de organización integradora.

Palabras clave: Participación de los estudiantes; Estructura organizativa colaborativa, Estrategias de aprendizaje, Aprendizaje colaborativo

\section{Summary}

The objective of this research was to determine the relationship between the participation of students, their learning strategies and the collaborative organization structure of the groups, with their learning of mathematics both individually and in groups, in a virtual group task of collaborative type, within the context of the health emergency generated by COVID19. The research design was cross-sectional descriptive non-experimental correlational. The study population was made up of 365 students from the Environmental Engineering program at the National University of Moquegua, with an intentional sample of 48 students enrolled in the Calculus I course, who formed 16 work groups made up of 3 members, who worked for 4 weeks, the resolution of 4 problematic situations related to the variation and change of functions. The information was delivered through the university platform and the group meeting was carried out through the WhatsApp tool. The results indicate that the improvement in the understanding, handling and translation of different representations of a mathematical object as well as the interpretation and manipulation of mathematical expressions has to do with the participation of the students within the virtual work group related to content of the task and that the groups where there is an improvement in learning to solve problems are those that develop an integrative organization structure

Keywords: Student participation; Collaborative organizational structure, Learning strategies, Collaborative learning

\section{Introducción}

A nivel universitario, el aprendizaje de la matemática representa un problema importante que se manifiesta por medio del alto número de estudiantes desaprobados como producto de una escasa interpretación de los diferentes conceptos que caracterizan a la matemática avanzada. Estas dificultades se ven favorecidas por la tendencia a la enseñanza tradicional por parte de los 
docentes así como la pasividad de los estudiantes al enfrentar su aprendizaje de tipo matemático.

La enseñanza habitual del cálculo no ha permitido un efectivo desarrollo del pensamiento matemático avanzado y ha centrado su accionar en el desarrollo de algoritmos de resolución de ejercicios tipo, por lo cual, si bien se puede enseñar a los estudiantes a realizar de forma más o menos mecánica algunos cálculos de derivadas y primitivas y a resolver algunos problemas estándar, se encuentran grandes dificultades para hacerlos entrar en verdad en el campo del cálculo y para hacerlos alcanzar una comprensión satisfactoria de los conceptos y métodos de pensamiento que son el centro de este campo de las matemáticas (Antigue, 1995; Cantoral; 2013).

La situación actual que se vive a nivel mundial como consecuencia de la emergencia sanitaria generada por el COVID 19 y las medidas de distanciamiento social emitidas, han desembocado que las universidades se vean obligadas a la construcción de una realidad caracterizada por la digitalización, donde el manejo de estrategias para la enseñanza y aprendizaje así como de las formas de trabajo de los estudiantes utilizadas de manera presencial, se han visto presionadas y reemplazadas por estrategias que caracterizan la educación a distancia (Vicentini, 2020), para las cuales muchos de los docentes y estudiantes no han estado preparados, poniendo en tela de juicio la efectividad de su actuación en el aprendizaje. En este contexto, se hace necesaria la evaluación de la forma de trabajo de los estudiantes sumergidos en esta nueva realidad en relación a su aprendizaje, y obtener a partir de ello información importante que permita proponer alternativas didácticas funcionales dentro de esta forma de trabajo caracterizada por la virtualidad y el trabajo remoto.

La presencia de las tecnologías de la información y la comunicación han venido generando en las universidades de corte tradicional un proceso de transformación importante, obligándolas al manejo de estrategias de instrucción caracterizadas por la interconexión de los estudiantes, en diferentes horarios y ubicaciones (Putnam, 2001). Estos nuevos espacios, adhieren a su propuesta actividades de aprendizaje colaborativo, ya que, según García (2014), las actividades en grupo generan procesos de demanda cognitiva como la resolución de conflictos, la argumentación y la indagación en comunidad. Se define la colaboración en un entorno virtual como un proceso compartido, coordinado e interdependiente, en el cual los estudiantes trabajan juntos para alcanzar un objetivo común en un entorno virtual (Guitert \& Pérez-Mateo, 2013)

Sfard (1988) planteó utilizar dos metáforas para explicar el aprendizaje colaborativo: como una adquisición individual del conocimiento y como una participación en una práctica social. En el primer caso, el aprendizaje se produce con la apropiación de conceptos que se genera en la discusión llevada a cabo durante una tarea colaborativa en un entorno virtual y que se produce al conectar los nuevos conocimientos con esquemas mentales preexistentes (Pozo, 1989). En cuanto a la segunda metáfora, el aprendizaje consiste en la participación y elaboración de conocimiento de manera conjunta, como el resultado de la interacción en una comunidad. Esta es una visión sobre el aprendizaje centrado en el proceso de conocer, más que en los resultados de conocimiento (García, 2014).

Dentro del marco de la primera metáfora para explicar el aprendizaje, se señalan cuatro elementos relevantes para el aprendizaje colaborativo en entornos virtuales por parte de estudiantes de educación superior: las características de la tarea, las características del grupo, las ayudas proporcionadas por el profesor y las estrategias de aprendizaje aplicadas por los estudiantes García (2014). En cuanto a las características de la tarea, el aprendizaje basado en la resolución de casos es una metodología de enseñanza que propicia un ambiente de instrucción para la participación activa de los estudiantes. La resolución de un caso exige de los estudiantes activar procesos para resolver problemas y adquirir una mejor comprensión de las situaciones trabajadas (Van Aalst, 2006). Otro tipo de tarea que utilizada también en el trabajo virtual es la elaboración colaborativa de proyecto, ya que ofrece la oportunidad de resolver problemas que se 
presentan en el mundo real (Thomas \& MacGregor, 2005). Estudios relacionados a este tipo de tareas en entornos virtuales reflejan su relación con el desarrollo de diferentes habilidades (Biasutti \& El-Deghaidy, 2012), quedando aún por investigar su relación con los resultados de aprendizaje.

Dentro de las características del grupo y su incidencia en el aprendizaje colaborativo en entornos virtuales, las investigaciones han considerado los siguientes elementos: su composición, distribución y su tamaño. Cuando se trabaja en entornos virtuales con grupos de trabajo colaborativo con más muchos integrantes, se obtienen calificaciones más bajas que en otros grupos con menos integrantes ya que en este último caso, aumenta la posibilidad de participación en los debates e interacción dentro del grupo, de manera que los estudiantes asumen la responsabilidad de su propio aprendizaje (Dewiyanti et al, 2007; Schelles \& Valcke, 2006). En cuanto a la asignación de roles dentro de los grupos virtuales de trabajo, permite involucrar de forma activa a los estudiantes con el contenido cognitivo y con los demás participantes. En la medida que se asignan roles dentro de los grupos virtuales de estudiantes universitarios, se mejoran los resultados de aprendizaje (Schelles \& Valcke, 2006)

Monereo (2000) defines las estrategias de aprendizaje como las acciones individuales, consientes e intencionales, que tienen la finalidad de cumplir con un objetivo de aprendizaje. Estas estrategias suelen agruparse en tres categorías: cognitivas que hacen alusión a las operaciones cognitivas para el procesamiento de la información; metacognitivas que hacen referencia a los procesos de planificación, control y evaluación por parte de los estudiantes; y las afectivas que tratan sobre las actitudes y motivaciones hacia el aprendizaje (Pintrich, 2000). ChanLin y Chan (2010) identificaron y agruparon estrategias manifestadas por estudiantes en un entorno virtual de aprendizaje en cuatro categorías: procesamiento de la información, coordinación y gestión de grupos, automonitorización y perfeccionamiento de la tarea, encontrando que algunas de estas estrategias correlacionan con los resultados de aprendizaje.

En el marco de la segunda metáfora para explicar el aprendizaje, se señalan tres características: la construcción social del conocimiento, la presencia cognitiva en el seno de una comunidad de indagación crítica y la construcción del conocimiento mediante la argumentación o el consenso alcanzado entre iguales (García, 2014). En el primer caso, el intercambio y negociación de ideas y conceptos es lo que genera el aprendizaje dentro de un entorno virtual de aprendizaje (Jonassen \& Land, 2000).

En cuanto a la segunda característica, los estudiantes construyen conocimiento por la presencia cognitiva que se genera en el seno de una comunidad de indagación crítica, en la interacción educativa dentro de un entorno virtual de aprendizaje (Garrinson et al, 2000). Además de ello, el éxito del aprendizaje en contexto de educación superior requiere la presencia del docente y la capacidad de proyectarse social y emocionalmente.

En cuanto a la tercera característica, se considera que los estudiantes aprenden socialmente cuando analizan diferentes perspectivas de resolución de un tema. En este marco, se sostiene que la discusión de argumentos genera conocimiento en el grupo. Además, dentro de un debate se construye conocimiento cuando se incorporan a las ideas propias las contribuciones de los demás (García, 2014). En esta línea, la valoración del tipo de participaciones de los estudiantes que estimulan el conocimiento constituye un elemento para determinar si se ha producido aprendizaje (Van Drie et al, 2005). Veerman y Veldhuis-Diermanse (2001) consideran importante diferenciar los mensajes de los estudiantes dentro los grupos virtuales en función de su propósito y el tipo de participación. También se considera importante valorar dentro de una discusión virtual, los mensajes en cuanto a su orientación al contenido, a lo técnico, a lo procedimental o no académicos (Van de Ven \& Poole, 2000).

La estructura organizativa colaborativa está referida al procedimiento que utilizan los estudiantes para coordinar y distribuirse el trabajo y desarrollar entre todos el informe final (Gar- 
cía, 2014). Una categorización de la organización de los grupos virtuales es propuesta por Engel y Onrubia (2010), quienes proponen lo siguiente: (1) la construcción paralela, donde cada miembro del grupo contribuye con una parte diferente de la tarea y el documento final era una yuxtaposición de estas partes, sin la contribución de los otros integrantes. (2) La construcción paralela, en la que cada miembro contribuye con un documento inicial, con la tarea completada en su totalidad o parcialmente, y el documento final se construía a través de la yuxtaposición de pequeñas piezas extraídas de las aportaciones iniciales de los otros integrantes. (3) La construcción secuencial sumativa donde un miembro del grupo presentaba un documento que constituía una propuesta inicial (parcial o completa) de resolución de la tarea, y el resto de los participantes agregaba sucesivamente sus contribuciones a este documento inicial, sin modificar lo escrito anteriormente, y por lo tanto, aceptando de manera sistemática lo que habían añadido otros miembros del grupo. (4) La construcción secuencial integradora donde un miembro del grupo presentaba un documento que constituía una propuesta de tarea inicial y los otros miembros del grupo contribuían sucesivamente a este documento, proponiendo modificaciones justificadas o discutiendo si estaban de acuerdo con lo que se había escrito anteriormente. (5) La construcción integradora, donde la redacción del documento se basa en una discusión sincrónica a través del chat, con revisiones, donde todos los miembros del grupo reaccionaban a los comentarios, los cambios y las aportaciones hechas por otros participantes.

Nuestro estudio centra su interés en la comprensión de la interacción de diferentes elementos individuales, del proceso social y la cognición resultante (Hakkinen, 2004). Por tal motivo, decidimos analizar, dentro del plano individual del proceso de aprendizaje colaborativo, las estrategias de aprendizaje de tipo cognitivo que aplican los estudiantes cuando realizan una tarea en grupo en un entorno virtual de trabajo; mientras que en plano del proceso de carácter social, analizamos las participaciones de los estudiantes en los grupos de trabajo y la estructura organizativa colaborativa y su relación con el aprendizaje tanto individual como grupal de la matemática.

\section{Método}

El presente estudio está enmarcado en un diseño no experimental transversal de tipo descriptivo correlacional que pretende encontrar la relación entre la participación de los estudiantes, sus estrategias de aprendizaje y la organización de los grupos con el aprendizaje de la matemática en estudiantes de educación superior, tanto individual como grupal, al desarrollar una tarea en grupos virtuales de trabajo. La población estuvo conformada por 365 estudiantes de la carrera profesional de Ingeniería Ambiental de la Universidad Nacional de Moquegua, de los cuales se tomó una muestra de tipo intencional conformada por 48 estudiantes matriculados en el curso de Cálculo I en el semestre 2020 - I, los cuales formaron 16 grupos de trabajo compuesto de 3 integrantes cada uno, donde uno de los estudiantes era el responsable de crear el grupo virtual a través de la herramienta WhatsApp, coordinar el trabajo con los integrantes del grupo y reportar al docente la información requerida a través de la plataforma virtual de la institución. Además, el docente también formaba parte de todos los grupos a fin de absolver alguna duda de los estudiantes. Cada grupo debió resolver 4 situaciones problémicas relacionadas al comportamiento variacional de las funciones en un lapso de 4 semanas.

\section{Instrumentos}

\section{Aprendizaje de la Matemática}

Para evaluar el rendimiento de los estudiantes se construyó y validó un cuestionario compuesto de 04 problemas relacionados al análisis variacional de las funciones: intervalos de crecimiento y decrecimiento, valores extremos y de estabilidad, intervalos de mayor crecimiento o decrecimiento, análisis del comportamiento variacional. Cada uno de estos problemas fue entregado a los grupos en forma semanal a través de la plataforma virtual de la institución, en la cual se es- 
tableció como tiempo de entrega una semana. El coeficiente de confiabilidad arrojó un valor de 0.82 , lo cual indica una alta confiabilidad.

Para evaluar el aprendizaje individual de cada estudiante, el cuestionario fue aplicado antes del inicio de los trabajos grupales y al final de ellos, comparándose estadísticamente los resultados obtenidos en cada proceso. Para la evaluación del aprendizaje grupal, se tomaron como referencia el promedio de los resultados obtenidos de cada reporte semanal de los grupos. En ambos casos, el aprendizaje de la matemática se evaluó a través de las siguientes dimensiones, propuestas por el Fondo de Investigación y Desarrollo en Educación (2011):

Tabla 1.

Dimensiones del aprendizaje de la Matemática

\begin{tabular}{ll}
\hline Dimensiones & Descriptores \\
\hline Resolución de Pro- & - Entender el problema \\
blemas & - Modelizar \\
& - Desarrollar y/ o adaptar estrategias para resolver problemas \\
& - Aplicar la estrategia para resolver el problema \\
& - Interpretar la respuesta en contexto del problema \\
& - Formular problemas \\
\hline Representación & Entender y utilizar las relaciones entre diversas representaciones de la \\
& - Escoger y traducir representaciones en otras \\
& - Usar representaciones para interpretar fenómenos físicos, sociales y \\
& matemáticos (construcción de modelo intermedio) \\
\hline Razonamiento y Ar- & - Formular, investigar conjeturas matemáticas a partir de regularidades \\
gumentación & - Sintetizar, sistematizar y generalizar conjeturas matemáticas \\
& - Elegir y utilizar varios tipos de razonamiento y demostración \\
& - Desarrollar y evaluar argumentos \\
\hline Cálculo y manipula- & - Comunicar su pensamiento matemático \\
ción de expresiones & - Usar y/o manipular expresiones matemáticas. \\
& - Calcular y/o cuantificar \\
& - Comunicar la manipulación de expresiones y cálculos \\
\hline
\end{tabular}

Fuente: Fondo de Investigación y Desarrollo en Educación (2011)

\section{Participación de los estudiantes}

En cuanto a la participación de los estudiantes, se utilizaron dos dimensiones de análisis: el Propósito de la Información y el Tipo de contenido, según las categorías propuestas por Veerman y Veldhuis-Diermanse (2001) y Lee et al (1999). Las categorías utilizadas para distinguir los diferentes mensajes de los estudiantes en los grupos de trabajos virtuales se muestran en la siguiente tabla:

Tabla 2.

Dimensiones de análisis de la participación de los estudiantes

\section{Categoría Descripción \\ Propósito de la Información}

Contenido Mensajes donde se identifican los asuntos y problemas relacionados con la tarea, se definen objetivos, se proporcionan referencias, enlaces, textos, se realizan preguntas de contenido, se dan valoraciones críticas y 
revisiones del contenido proporcionado.

Organización Mensajes sobre la organización del tiempo y la tarea, propuestas de actividades en relación con el trabajo, la distribución de roles o tareas, la coordinación y

confirmación de la gestión del trabajo, llamadas de atención a cumplir con la organización acordada

Social Mensajes relacionadas con presentaciones personales (quiénes son, qué hacen, dónde trabajan), temas familiares y problemas personales de todo tipo, incluidos técnicos.

\section{Tipo de Contenido}

Idea Nueva

Mensaje orientado a la tarea, focalizado en un contenido relevante, no mencionado con anterioridad

Ampliación Mensaje cuya idea redefine o amplia de forma elaborada algo dicho con anterioridad

Valoración Aportación crítica sobre algo aportado por otro compañero que supone un razonamiento o justificación (no un simple si/no)

Repetición Aquellos mensajes que no se adhieren a las categorías anteriores

Preguntas Son mensajes que, además de pertenecer a alguna de las categorías anteriores, contienen una o más preguntas relacionadas con el contenido del trabajo.

Las preguntas pueden ser básicamente de tres tipos: clarificar una duda, obtener una información ampliada de algún tema o pedir a los compañeros la confirmación sobre un contenido ya tratado.

Explicaciones Son mensajes que ofrecen respuesta cualquiera de las preguntas planteadas, identificadas en la categoría anterior. No es un simple si/no, ya que debe contener una explicación de la respuesta dada.

Fuente: Veerman y Veldhuis-Diermanse (2001)

Los mensajes de los estudiantes fueron categorizados en cada una de las 8 reuniones grupales llevadas a cabo. Las frecuencias obtenidas en cada categoría fueron sistematizadas a través de un archivo Excel, obteniéndose al final un puntaje en cada una de ellas. La suma de los puntajes finales en cada una de las categorías definió el resultado de la participación de los estudiantes.

\section{Estructura Organizativa Colaborativa}

Para el análisis de la Estructura de Organización Colaborativa, se utilizó la propuesta de Engel y Onrubia (2010) quienes encontraron tres tipos básicos de estructura colaborativa, las cuales se muestran en la siguiente tabla:

Tabla 3.

Tipo de Estructura Organizativa Colaborativa

\begin{tabular}{ll}
\hline Categoría & Definición \\
\hline Sumativa & Los estudiantes deciden distribuir la tarea entre todos los miembros y desa- \\
& rrollar el trabajo uniendo diferentes piezas elaboradas de forma independien- \\
& tes por cada integrante. El documento final es básicamente una suma de las \\
& partes diferenciadas por pocas revisiones de los miembros y prácticamente
\end{tabular}


ningún feedback final ni pregunta entre ellos

Agregadora Los estudiantes aprueban un texto inicial (habitualmente proporcionado por uno de ellos), al que se va agregando contribuciones parciales de los miembros del grupo y finalmente realizan una revisión del documento final entre ellos, con un feedback escaso

Integradora Los estudiantes deciden contribuir prácticamente a todas las secciones del informe escrito y trabajan conjuntamente con el texto a partir de una interacción, revisión y feedback de los textos escritos de forma integrada. Prácticamente todo el grupo proporciona feedback y el texto final recoge las revisiones de todos

Fuente: Engel y Onrubia (2010)

La valoración de la estructura organizativa utilizada por cada grupo se hizo en base a las cinco categorías propuestas por García (2014):

(1) Contribución igualitaria al trabajo, referida a la forma de distribuir el trabajo, siendo alta cuando todos los miembros aportan a todas las partes del trabajo y baja cuando los miembros elaboran el trabajo en piezas separadas.

(2) Asignación de responsabilidades, referido al grado de reparto del contenido, siendo bajo si se nombraba a un miembro responsable de una parte y sólo una, y alto, si todos se responsabilizaban del resultado de todas las partes del informe.

(3) Reciprocidad, hace referencia a la aceptación de las propuestas de los compañeros. El extremo más bajo implica poca aceptación y por tanto, escasa modificación posterior del contenido y el extremo alto implica aceptación y valoración positiva, y por tanto, integración de las aportaciones de todos los miembros del grupo.

(4) La revisión del trabajo final hace referencia al grado (alto o bajo) en que los miembros del grupo revisan, valoran y aportan al resultado final del trabajo escrito.

(5) El grado de consenso puede ser alto si casi todos los miembros del grupo han manifestado sus acuerdos y posibles desacuerdos y han llegado a un consenso, y bajo si no han realizado casi manifestaciones al respecto o no han alcanzado un consenso.

Los grupos con los niveles inferiores en casi todas las categorías habrían utilizado una estructura colaborativa sumativa, los grupos con los niveles más altos una estructura colaborativa integradora, y los grupos con niveles intermedios una estructura colaborativa agregadora. Otorgamos un valor de 5 al nivel alto en cada categoría y un valor de 1 al nivel bajo. Los grupos que obtuvieran una puntuación total entre 5 y 11 puntos la categoría de estructura colaborativa sumativa, a los que obtuvieran una puntuación total de entre 12 y 18 puntos, la categoría de estructura colaborativa agregadora y, finalmente a los grupos que obtuvieran una puntuación total de entre 19 y 25 puntos, la categoría de estructura colaborativa integradora. Luego de analizar las participaciones de los estudiantes, categorizamos sus intervenciones en una ficha otorgando un valor a cada grupo en cada categoría. Finalmente, la suma de valores resultante nos dio la estructura colaborativa aplicada por cada grupo.

\section{Estrategias de Aprendizaje}

En cuanto a las estrategias de aprendizaje de los estudiantes, se siguió la categorización realizada por García (2014): 
Tabla 4.

Estrategias de aprendizaje

\begin{tabular}{|c|c|c|}
\hline Estrategia & Definición & Acciones \\
\hline $\begin{array}{l}\text { Buscar } \\
\text { información }\end{array}$ & $\begin{array}{l}\text { Actividades que hace } \\
\text { un } \\
\text { estudiante para } \\
\text { encontrar la informa- } \\
\text { ción } \\
\text { que necesita para apor- } \\
\text { tar } \\
\text { contenido al texto del } \\
\text { informe final del grupo. }\end{array}$ & $\begin{array}{l}\text { - Buscar información. } \\
\text { - Encontrar y recoger información de Internet. } \\
\text { - Buscar en websites, foros, artículos y enlaces, }\end{array}$ \\
\hline $\begin{array}{l}\text { Compartir } \\
\text { información }\end{array}$ & $\begin{array}{l}\text { Actividades que } \\
\text { implican compartir o } \\
\text { intercambiar la } \\
\text { información elaborada } \\
\text { por uno mismo o } \\
\text { encontrada con los } \\
\text { compañeros del grupo } \\
\text { por medio de texto } \\
\text { escrito (mail, foro). }\end{array}$ & $\begin{array}{l}\text { - Remitir mensajes con información externa en- } \\
\text { contrada: urls, libros, artículos. } \\
\text { - Remitir mensajes con información de contenido } \\
\text { trabajada por el estudiante: avance del trabajo, } \\
\text { comentarios críticos y opiniones, observaciones } \\
\text { y sugerencias, ideas y conclusiones (tanto dentro } \\
\text { de un mensaje como en un fichero adjunto). }\end{array}$ \\
\hline $\begin{array}{l}\text { Leer } \\
\text { contenidos y } \\
\text { documentación }\end{array}$ & $\begin{array}{l}\text { Actividades que } \\
\text { implican una selección } \\
\text { de la documentación } \\
\text { encontrada o recibida, } \\
\text { así como una lectura } \\
\text { superficial de la } \\
\text { información. }\end{array}$ & $\begin{array}{l}\text { - Revisar y leer la literatura encontrada (enlaces, } \\
\text { libros, artículos). } \\
\text { - Leer información proporcionada por el profesor } \\
\text { - Leer la información proporcionada por otro estu- } \\
\text { diante. } \\
\text { - Leer los textos escritos por otros estudiantes. }\end{array}$ \\
\hline $\begin{array}{l}\text { Leer los } \\
\text { mensajes del } \\
\text { WhatsApp }\end{array}$ & $\begin{array}{l}\text { Actividades que consis- } \\
\text { ten en leer lo que } \\
\text { otros compañeros han } \\
\text { escrito en el foro. }\end{array}$ & $\begin{array}{l}\text { Leer los mensajes escritos por los compañeros en } \\
\text { el WhatsApp. } \\
\text { Leer los mensajes del profesor. }\end{array}$ \\
\hline $\begin{array}{l}\text { Analizar la } \\
\text { información }\end{array}$ & $\begin{array}{l}\text { Actividades que } \\
\text { implican evaluar, } \\
\text { comparar, discriminar, } \\
\text { seleccionar o resumir } \\
\text { información de } \\
\text { contenido relevante al } \\
\text { texto del informe del } \\
\text { grupo. }\end{array}$ & $\begin{array}{l}\text { - Analizar y seleccionar la información relevante } \\
\text { de fuentes externas (enlaces, webs, artículos). } \\
\text { - Analizar y seleccionar la información relevante } \\
\text { de fuentes internas (textos es por compañeros). } \\
\text { - Evaluar y resumir la información para extraer } \\
\text { notas, ideas, conclusiones y contribuciones al in- } \\
\text { forme. }\end{array}$ \\
\hline
\end{tabular}

\begin{tabular}{llll}
\hline $\begin{array}{l}\text { Redactar } \\
\text { información }\end{array}$ & la & $\begin{array}{l}\text { Escribir y redactar de } \\
\text { acuerdo a la estructura } \\
\text { del informe, los } \\
\text { contenidos elaborados } \\
\text { previamente por el } \\
\text { estudiante. }\end{array}$ & $\begin{array}{l}\text { - Escribir y componer los textos relacionados con } \\
\text { el informe. }\end{array}$ \\
& $\begin{array}{l}\text { - Desarrollar los elementos adicionales que com- } \\
\text { plementan el texto (gráficos imágenes, dibujos) }\end{array}$ & - Realizar correcciones a los borradores del grupo. \\
& $\begin{array}{l}\text { Acciones no listadas en } \\
\text { las categorías 1 a } 6 .\end{array}$ & $\begin{array}{l}\text { Actividades de organización personal y activida- } \\
\text { des mecánicas como imprimir, subir y bajar fiche- } \\
\text { Otras al campus virtual. }\end{array}$
\end{tabular}

Fuente: García (2014) 
Al inicio de cada reunión en el WhatsApp el docente pedía a cada integrante del grupo que describiera si había buscado en internet o en otro medio alguna información relacionada al tema. Luego, se revisaron las conversaciones efectuadas por los estudiantes en el grupo de WhatsApp a fin de identificar las estrategias que utilizaban e ir anotando la frecuencia de uso en cada una de las reuniones programadas en una ficha. Al final, se sumaron todas las frecuencias obtenidas para obtener el puntaje por cada una de las estrategias utilizadas.

\section{Análisis de Datos}

La determinación de las correlaciones entre el aprendizaje de la matemática, tanto a nivel individual como grupa, con la participación de los estudiantes y las estrategias de aprendizaje se realizó mediante del Coeficiente de Correlación de Spearman, ya que los datos obtenidos no seguían una distribución normal. Además, se utilizó la Prueba no paramétrica de Kruskal-Wallis con la finalidad de determinar diferencias en las medias de los diferentes tipos de estructuras organizacionales colaborativas y el aprendizaje grupal. Todos estos análisis se efectuaron en el programa estadístico SPSS 22 a un nivel de significancia de 0.05 y 0.01 .

\section{Resultados}

A continuación, se presentan los resultados obtenidos en el análisis de relación entre las variables de estudio: participación de los estudiantes, estrategias de aprendizaje y estructura organizacional colaborativa con el aprendizaje de la matemática, en su faceta individual y grupal.

\section{Relación entre participación de los estudiantes y el aprendizaje de la matemática}

Tabla 5.

Participación de los estudiantes según el propósito de la información y aprendizaje individual de la matemática

\begin{tabular}{lllllllll}
$M$ & $\mathrm{DE}$ & $(1)$ & $(2)$ & $(3)$ & $(4)$ & $(5)$ & (6) & (7) \\
\hline
\end{tabular}

\begin{tabular}{|c|c|c|c|c|c|c|c|c|}
\hline \multicolumn{9}{|l|}{ Aprendizaje de la Matemática } \\
\hline (1) Resolución de Problemas & 3.06 & 0.78 & - & & & & & \\
\hline (2) Representación & 2.65 & 0.56 & $0.48 *$ & - & & & & \\
\hline \multirow{2}{*}{$\begin{array}{l}\text { (3) Razonamiento y argumentación } \\
\text { (4) Cálculo y manipulación de expre- } \\
\text { siones }\end{array}$} & 2.60 & 0.54 & $0.42 *$ & $0.51^{*}$ & - & & & \\
\hline & 2.83 & 0.38 & $0.45^{*}$ & $0.49 *$ & $0.44 *$ & - & & \\
\hline \multicolumn{9}{|l|}{ Propósito de la Información } \\
\hline (5) Contenido & 7.80 & 0.74 & $0.32 * *$ & $0.23 *$ & $0.38 * *$ & $0.35 *$ & - & \\
\hline (6) Organización & 6.63 & 0.65 & 0.21 & 0.34 & 0.11 & 0.31 & $0.17 *$ & - \\
\hline (7) Social & 2.06 & 0.28 & -0.18 & -0.21 & -0.14 & -0.19 & -0.22 & 0.24 \\
\hline
\end{tabular}

$* \mathrm{p}<0.05, * * \mathrm{p}<0.01$

Los resultados encontrados en la tabla muestran relación moderada, positiva muy significativa entre las participaciones orientadas al contenido y la resolución de problemas $(\mathrm{r}=0.32, \mathrm{p}<$ $0.01)$, así como con la faceta de razonamiento y argumentación $(\mathrm{r}=0.38, \mathrm{p}<0.01)$. De manera similar, este tipo de participaciones a nivel individual tienen relación moderada, positiva significativa con la representación $(\mathrm{r}=0.23, \mathrm{p}<0.05)$ y con el cálculo y manipulación de expresiones $(r=0.35, p<0.05)$ realizadas por los estudiantes. En tal sentido, dentro del trabajo en grupo realizado de manera virtual por los estudiantes, se constata que mientras se expresen mayor cantidad de mensajes que permitan definir objetivos de trabajo, proporcionar referencias y expresar valoraciones críticas y revisiones del contenido proporcionados en algún momento de la tarea, se genera un mejor aprendizaje de la matemática, sobre todo en aquellos aspectos relacionados a 
la resolución de problemas y al razonamiento y argumentación, que permiten entender y plantear estrategias para dar solución a un problema, como a la formulación de conjeturas y el desarrollo y evaluación de argumentos.

De manera similar, la mejora en el entendimiento, manejo y traducción de distintas representaciones de un objeto matemático, así como de la interpretación y manipulación de las expresiones matemáticas tiene que ver con las participaciones de los estudiantes dentro del grupo virtual de trabajo relacionadas con algún asunto del contenido de la tarea que deben dar solución de manera grupal.

Tabla 6.

Participación de los estudiantes según el tipo de contenido y aprendizaje individual de la matemática

\begin{tabular}{|c|c|c|c|c|c|c|c|c|c|c|c|c|}
\hline & $\mathbf{M}$ & DE & (1) & (2) & (3) & (4) & (5) & (6) & (7) & (8) & (9) & (10) \\
\hline $\begin{array}{l}\text { Aprendizaje de la Ma- } \\
\text { temática } \\
\text { (1) Resolución de Pro- } \\
\text { blemas }\end{array}$ & 3.06 & 0.78 & '- & & & & & & & & & \\
\hline $\begin{array}{l}\text { (2) Representación } \\
\text { (3) Razonamiento y ar- } \\
\text { gumentación } \\
\text { (4) Cálculo y manipula- } \\
\text { ción de expresiones }\end{array}$ & $\begin{array}{l}2.65 \\
2.60 \\
2.83\end{array}$ & $\begin{array}{l}0.56 \\
0.54\end{array}$ & $\begin{array}{l}0.48 * \\
0.42 *\end{array}$ & $\begin{array}{l}- \\
0.51 * \\
0.49 *\end{array}$ & $\begin{array}{l}- \\
0.44 *\end{array}$ & & & & & & & \\
\hline
\end{tabular}

\section{Tipo de Contenido}

(5) Idea Nueva

(6) Ampliación

$\begin{array}{lllllll}8.8 & 1.2 & 0.54 * * & 0.31 & 0.38 * & 0.27 & -\end{array}$

(7) Valoración

$\begin{array}{llllllll}6.9 & 0.8 & 0.32 * & 0.22 & 0.41 * & 0.18 & 0.34 *\end{array}$

(8) Repetición

$\begin{array}{llllllllll}5.8 & 0.7 & 0.17 & 0.25 & 0.33 * & 0.37 & 0.26 * & 0.28 * & -\end{array}$

(9) Preguntas

$\begin{array}{llll}6.6 & 0.4 & -0.11 & 0.33\end{array}$

$\begin{array}{llllll}0.16 & 0.28 & 0.14 & 0.19 & 0.29\end{array}$

(10) Explicaciones

$\begin{array}{llllllllll}6.0 & 0.6 & 0.19 & 0.28 & 0.21 & 0.34 & 0.47 * & 0.38 & 0.36 & 0.17\end{array}-$

$* \mathrm{p}<0.05, * * \mathrm{p}<0.01$

En cuanto a la participación de los estudiantes según el tipo de contenido, se encontró que las intervenciones que expresan ideas nuevas $(\mathrm{r}=0.54 \mathrm{p}<0.01)$ y las que buscan explicar alguna pregunta formulada por algún integrante del grupo $(\mathrm{r}=0.51, \mathrm{p}<0.01)$, tienen relación alta, positiva y muy significativa con la resolución de problemas. De manera moderada, los mensajes que amplían ideas mencionadas por algún integrante del grupo virtual de trabajo, también relacionan significativamente con la resolución de problemas $(\mathrm{r}=0.32, \mathrm{p}<0.05)$ y con el razonamiento y argumentación $(\mathrm{r}=0.41, \mathrm{p}<0.05)$. También, la expresión de ideas nuevas $(\mathrm{r}=0.38 \mathrm{p}$ $<0.05)$ y la valoración de las aportaciones de otros compañeros $(\mathrm{r}=0.33 . \mathrm{p}<0.05)$ se relacionan moderada, positiva y significativamente con el razonamiento y argumentación, quien a su vez presenta una baja, positiva y significativa relación con las explicaciones realizadas a alguna pregunta formulada.

En tal sentido, la mejora en el entendimiento de un problema, así como el desarrollo y aplicación de estrategias de resolución tienen que ver con una mayor expresión de mensajes que centran su interés en algún contenido relevante de la tarea así como en aquellos que buscan aclarar alguna duda o interrogante; y con menor intensidad con aquellos mensajes que buscan ampliar de manera más elaborada algún mensaje anterior. La formulación de conjeturas, la elección y uso de diversos tipos de razonamiento, así como el desarrollo de argumento también se ven favorecidos con una mayor expresión de mensajes orientados directamente en la tarea, los que redefinen algún dicho anterior y los que expresan una aportación crítica a algún aporte de otro 
compañero, y con menor intensidad, por aquellos mensajes que buscan plantear una explicación a alguna pregunta formulada por algún integrante del grupo.

Tabla 7.

Participación de los estudiantes según el propósito de la información y aprendizaje grupal de la matemática.

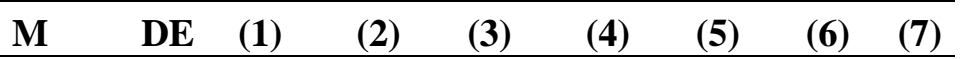

\section{Aprendizaje de la Matemática}

(1) Resolución de Problemas

$3.250 .39 '-$

(2) Representación

$\begin{array}{llll}2.86 & 0.20 & 0.51 * & -\end{array}$

(3) Razonamiento y argumentación

$2.28 \quad 0.20 \quad 0.48 * 0.41 *$

(4) Cálculo y manipulación de expresiones

$\begin{array}{llllll}3.08 & 0.33 & 0.38^{*} & 0.35^{*} & 0.49^{*}-\end{array}$

\section{Propósito de la Información}

(5) Contenido

(6) Organización

(7) Social $* \mathrm{p}<0.05$
$62.44 \quad 5.88 \quad 0.42 * \quad 0.38 * 0.33 * 0.25 *$ -

$\begin{array}{llllllll}53.00 & 5.32 & 0.33 & 0.26 & 0.27 & 0.38 & 0.21 * & -\end{array}$

$\begin{array}{llllllllll}16.50 & 2.23 & -0.31 & -0.11 & -0.29 & -0.17 & -0.19 & 0.31 & -\end{array}$

En cuanto al aprendizaje grupal, la tabla anterior muestra que los mensajes intercambiados en el grupo que expresan contribuciones de contenido para el trabajo en que se está colaborando y que deben resolver en grupo, se relaciona moderada, positiva y significativamente con todas las dimensiones del aprendizaje de la matemática $(\mathrm{r}<0.5, \mathrm{p}<0.05)$. En tal sentido, la mejora en la resolución de problemas, en el manejo de diversas representaciones, en el uso de diferentes tipos de razonamiento y el desarrollo de argumentos así como la interpretación y manipulación de expresiones matemáticas se ve moderadamente favorecido cuando en el grupo de trabajo se genera un mayor intercambio de mensajes que definen objetivos para la realización de la tarea, donde se efectúen preguntas de contenido y se expresen valoraciones críticas así como revisiones del contenido proporcionado.

\section{Relación entre estructura organizativa colaborativa y aprendizaje de la matemática}

Tabla 8.

Aprendizaje grupal de la matemática según la estructura organizativa colaborativa.

\begin{tabular}{lllllll}
\hline \multirow{2}{*}{ Aprendizaje de la Matemática } & \multicolumn{2}{l}{$\begin{array}{l}\text { Sumativa } \\
(\mathbf{3 8 \%})\end{array}$} & \multicolumn{3}{l}{$\begin{array}{l}\text { Agregadora } \\
\mathbf{( 3 1 \% )}\end{array}$} & \multicolumn{3}{l}{$\begin{array}{l}\text { Integradora } \\
(\mathbf{3 1 \% )})\end{array}$} \\
& $\mathrm{M}$ & $\mathrm{DE}$ & $\mathrm{M}$ & $\mathrm{DE}$ & $\mathrm{M}$ & $\mathrm{DE}$ \\
\hline Resolución de Problemas & 2.93 & 0.12 & 3.20 & 0.33 & 3.44 & 0.43 \\
Representación & 2.93 & 0.12 & 2.95 & 0.11 & 2.94 & 0.12 \\
Razonamiento y argumentación & 2.29 & 0.22 & 2.45 & 0.33 & 2.44 & 0.38 \\
Cálculo y manipulación de expresiones & 2.93 & 0.12 & 3.00 & 0.18 & 3.06 & 0.24 \\
\hline
\end{tabular}

La tabla anterior muestra que $38 \%$ de los grupos virtuales de trabajo muestran una estructura organizativa donde el trabajo final es la suma de partes desarrolladas de forma independiente con poca revisiones por parte de los integrantes del grupo y con ausencia de feedback, otro $31 \%$ de los grupos virtuales muestra una estructura de organización donde los integrantes van agregando contribuciones parciales y efectúan una revisión del trabajo final entre ellos y el $31 \%$ de grupos virtuales restantes muestra una estructura de organización donde los integrantes van contribuyendo en todas las partes del trabajo final existiendo una revisión por parte de todos los integrantes del grupo. En este último tipo de grupos de trabajo virtuales se refleja las mayores 
medias en las diferentes dimensiones del aprendizaje de la matemática del grupo. Aun así, el resultado del análisis de diferencia de medias mediante la prueba de Kruskal-Wallis indica que existen diferencias significativas en función de la estructura de organización colaborativa integradora en relación a la resolución de problemas $[X 2=5,04 ; \mathrm{p}<0,05]$ pero no en el aprendizaje de las representaciones $[X 2=5,97 ; p=0,121]$, del razonamiento y argumentación $[X 2=6,02 ; p$ $=0,066]$ y del cálculo y manipulación de expresiones $[X 2=6.12 ; \mathrm{p}=0,063]$.

En tal sentido, el trabajo de grupos virtuales que se caracterizan por la contribución permanente de sus integrantes en todas las etapas de desarrollo de la tarea propuesta efectuada a través de la interacción, revisión y feedback de los textos generados de forma integrada, favorece un mejor entendimiento del problema propuesto, permitiendo el desarrollo y aplicación de estrategias de resolución.

\section{Relación entre las estrategias de aprendizaje y aprendizaje individual de la matemática}

Tabla 9.

Estrategia de aprendizaje y aprendizaje individual de la matemática

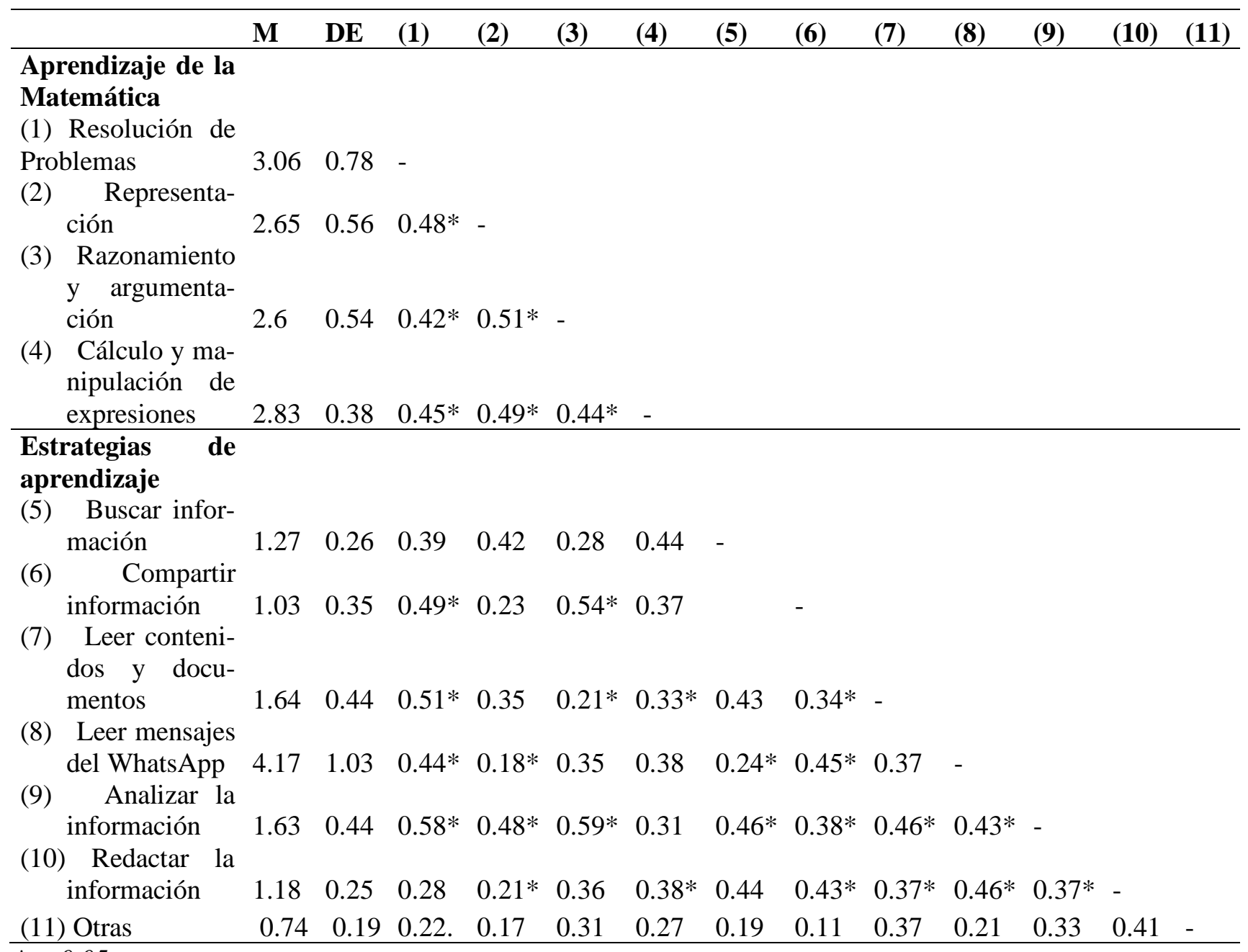
$* p<0.05$

La tabla muestra que el aprendizaje de los estudiantes sobre la resolución de problemas está relacionado alta, positiva y significativamente con la lectura de contenidos y documentos $(\mathrm{r}=0.51, \mathrm{p}<0.05)$ y el análisis de la información $(\mathrm{r}=0.58, \mathrm{p}<0.05)$, mientras que la relación es moderada con la lectura de los mensajes realizados en el grupos de WhatsApp $(r=0.44$, $\mathrm{p}<0.05)$ y con el compartir la información $(\mathrm{r}=0.49, \mathrm{p}<0.05)$. En cuanto al aprendizaje de las 
representaciones, existe relación moderada, positiva y significativa $(r=0.48, p<0.05)$ con el análisis de la información, mientras que la relación es baja con la lectura de mensajes de whassap $(\mathrm{r}=0.18, \mathrm{p}<0.05)$ y la redacción de la información $(\mathrm{r}=0.21, \mathrm{p}<0.05)$. El aprendizaje del razonamiento y argumentación se relaciona alta, positiva y significativamente con el análisis de la información $(\mathrm{r}=0.59, \mathrm{p}<0.05)$ y con el compartir la información $(\mathrm{r}=0.54, \mathrm{p}<0.05)$, mientras que la relación es moderada con la lectura de contenidos y documentación $(r=0.21, p<$ 0.05). También se encontró relación moderada, positiva y significativa entre el aprendizaje del cálculo y manipulación de expresiones matemáticas con la lectura de contenidos y documentos $(\mathrm{r}=0.33, \mathrm{p}<0.05)$ y la redacción de documentación $(\mathrm{r}=0.38, \mathrm{p}<0.05)$.

En tal sentido, un mayor intercambio de información construida por los integrantes del grupo o encontrada en otros medios, favorece la comprensión del problema y la generación de estrategias de resolución, así como el uso de diferentes tipos de razonamiento y desarrollo de argumento. Una mayor selección de documentación, así como su lectura favorece también la generación de estrategias de resolución de problemas y con menor intensidad, la interpretación y uso de expresiones matemáticas, así como desarrollo de argumentos y formas de razonamiento. Una mayor lectura de lo que los integrantes del grupo expresan en el WhatsApp también contribuye a la resolución de problemas y en menor medida al manejo y comprensión de las diferentes formas de representación de un objeto matemático. El incremento de la evaluación comparación y discriminación de la información que se escribe o comparte en el grupo WhatsApp de trabajo favorece la resolución de problemas, el manejo de representaciones y la generación de argumentos y diversos tipos de razonamiento. La interpretación y uso de expresiones matemáticas y el manejo de formas de representación se ve favorecida cuando se escribe o redacta los contenidos elaborados previamente por los integrantes del grupo.

\section{Discusión}

El presente estudio tuvo como objetivo fue determinar la relación entre la participación de los estudiantes, sus estrategias de aprendizaje y la estructura de organización colaborativa de grupos de trabajo virtuales, con su aprendizaje de la matemática tanto a nivel individual como grupal, en una tarea virtual de tipo colaborativa, desarrollada en el contexto de la emergencia sanitaria generada por el COVID19.

Los resultados obtenidos luego del análisis de correlación efectuado reflejan la relación que existe entre algunos tipos de participación de los estudiantes y su aprendizaje de la matemática, tanto a nivel individual como grupal. Se constata que las participaciones de los estudiantes que tienen que ver con la definición de objetivos de trabajo para la realización de la tarea están relacionadas con la mejora del aprendizaje de la matemática en aspectos relacionados a la resolución de problemas, el desarrollo de argumentos y tipos de razonamiento, el manejo de representaciones y la interpretación y manejo de expresiones matemáticas, reflejando que en el trabajo de grupos virtuales, el aprendizaje de tipo colaborativo tiene conexión con las participaciones de contenido (García, 2014).

La generación de mensajes orientados directamente en la tarea y los que buscan profundizar de forma crítica en alguna idea expresada con anterioridad, reflejan el carácter dinámico del trabajo grupal de los estudiantes. Esta controversia en la interacción de los estudiantes contribuye al aprendizaje de los procedimientos, fórmulas y conceptos a considerar en la resolución de problemas matemáticos (Rojas, Carretero \& Alvarez, 2012) y a su vez generan en los estudiantes la necesidad de construir razonamientos tendientes a la resolución de problemas (Zuloaga, Pérez \& Gómez, 2014).

El aprendizaje de matemática en los estudiantes se ve favorecido en la medida en que en la interacción virtual de los estudiantes en los grupos de trabajo, se produzcan mensajes orientados al desarrollo de la tarea o que buscan redefinir o ampliar una idea de algún compañero o dar 
una respuesta elaborada a alguna duda. Esta necesidad de buscar compartir las ideas propias con otras y poner a discusión de los otros integrantes tiene conexión con la construcción individual del conocimiento y de la elaboración conceptual propia (Van Boxtel et al, 2000), sin embargo, a diferencia de los señalado por Chin y Osborne (2010), la formulación de interrogantes por parte de los estudiantes no ha incidido en el desarrollo de argumentos y formas de razonamiento, y en general, en el aprendizaje de la matemática.

La forma de trabajo grupal donde se refleja el aporte permanente de todos los estudiantes para la construcción del informe final de la tarea propuesta incide de manera significativa en el desarrollo de estrategias de resolución de problemas, sea a nivel individual como grupal, con lo cual, la estructura de organización que exigen una mayor colaboración de los estudiantes mejora los procesos de construcción de conocimiento (García, 2014) y pone de manifiesto que, el conocimiento propio que es discutido en grupo, motiva la construcción de nuevo conocimiento (Scardamalia \& Bereiter, 1992).

Por otro lado, los resultados obtenidos muestran que las actividades de los estudiantes que tienen que ver con el intercambio de información propia u obtenida por otros estudiantes, así como aquellas que involucran la comparación y análisis de contenido relevante para el desarrollo de la tarea tiene conexión con la mejora del aprendizaje de la matemática, en aspectos relacionados a la resolución de problemas, manejo de representación y desarrollo de argumentos y formas de razonamientos. Esta incidencia en el aprendizaje coincide con lo señalado por ChanLin y Chan (2010) y lo repostado por García (2014) quienes sostienen que el tratamiento, coordinación y gestión de la información se relacionan con los logros de aprendizaje. La lectura de información compartida ya sea a través de herramientas informáticas como el whassap o de documentación proporcionada por otros estudiantes dentro del grupo así como la redacción y composición de textos relacionados al desarrollo de la tarea constituyen habilidades de interacción social y aprendizaje con sus compañeros y para el manejo de recursos para usar la información obtenida, lo ha incidido significativamente en el aprendizaje de la matemática, coincidiendo con lo encontrado por Chávez (2018).

\section{Referencias}

Artigue, M. (1995). La enseñanza de los principios del cálculo: problemas epistemológicos, cognitivos y didácticos. En Artigue, M.; Douady, R.; Moreno, L.; Gómez, P., (Eds.). Ingeniería didáctica en educación matemática (pp. 97-140). México: Grupo Editorial Iberoamérica. Recuperado de https://es.scribd.com/document/262352365/La-ensenanza-delos-principios-del-calculo-problemas-epistemologicos-cognitivos-y-didacticos

Biasutti, M., \& El-Deghaidy, H. (2012). Using Wiki in teacher education: Impact on knowledge management processes and student satisfaction. Computers \& Education, 59(3), 861-872. doi: https://doi.org/10.1016/j.compedu.2012.04.009

Cantoral, R. (2013). Desarrollo del Pensamiento y Lenguaje Variacional. México: Progreso S. A.

ChanLin, L. \& Chan, K. (2010) Group learning strategies for online course. Procedia - Social and Behavioral Sciences, 2(2), 397-401. doi: https://doi.org/10.1016/j.sbspro.2010.03.033.

Chávez, A., Leticia (2018). Estrategias de Aprendizaje y Rendimiento Académico en la asignatura de Análisis Matemático II. Revista Educación XXVII. (53) p. 24-40. doi: https://doi.org/10.18800/educacion.201802.002

Chin, C. \& Osborne, J. (2010) Students' questions and discursive interaction: Their impact on argumentation during collaborative group discussions in science. Journal of Research in Science Teaching, 47 (7), 883-908. doi: https://doi.org/10.1002/tea.20385

Dewiyanti, S., Brand-Gruwel, S., Jochems, W., \& Broers, N. (2007). Students experiences with collaborative learning in asynchronous computer-supported collaborative learning environments. Computers in Human Behavior, 23, 496-514. doi: https://doi.org/10.1016/j.chb.2004.10.021 
Engel, A \& Onrubia, J (2010). Patrones de organización grupal y fases de construcción del conocimiento en entornos virtuales de aprendizaje colaborativo. Infancia y Aprendizaje, 33 (4), 515-528. doi: 10.1174/021037010793139608

García, C. (2014). Aprendizaje colaborativo en grupos virtuales. Tesis Doctoral. Universitat Oberta de Catalunya. España. Recuperado de: https://www.tesisenred.net/bitstream/handle/10803/307052/Tesis\%20Consuelo\%20Garci a\%20Tamarit.pdf? sequence $=1 \&$ isAllowed $=y$

Garrison, D.R., Anderson, T., \& Archer, W. (2000). Critical Inquiry in a Text-Based Environment: Computer Conferencing in Higher Education. The Internet and Higher Education 2, 2-3, 87-105. doi: https://doi.org/10.1016/S1096-7516(00)00016-6

Guitert, M., \& Pérez-Mateo, M. (2013). La colaboración en la red: hacia una definición de aprendizaje colaborativo en entornos virtuales. Teoría de la Educación. Educación y Cultura en la Sociedad de la Información, 14(1), 10-31. ISO 690. doi: http://dx.doi.org/10.14201/eks.9440

Häkkinen, P. (2004). What makes learning and understanding in virtual teams so difficult?. CyberPsychology \& behavior, 7(2), 201-206. doi: 10.1089/109493104323024465

Jonnasen, D. \& Land, S. (2000). Theoretical Foundations of Learning Environments. Lawrence Eribaum. Recuperado de: https://www.researchgate.net/publication/237035484_Theoretical_Foundations_of_Learn ing_Environments

Lee, F-L., Liang, S. \& Cahn, T-W (1999). An attempt to design synchronouscollaborative learning environments for peer dyads on the world wide web. Journalof Educational Computing Research, 21(2) 221-253. doi: https://doi.org/10.2190/J9V5-DVA1-5FLPUE3M

Monereo, C. (2000). El asesoramiento en el ámbito de las estrategias de aprendizaje. En C. Monereo (Coord.), Estrategias de aprendizaje (pp. 15-62). Madrid: Visor

Pintrich, P. R. (2000). The role of goal orientation in self-regulated learning. En M. Boekaerts, P. R. Pintrich, \& M. Zeidner (Eds.), Handbook of self-regulation: Theory, research and applications (pp. 452-502). San Diego, CA: Academic Press. doi: https://doi.org/10.1016/B978-012109890-2/50043-3

Pozo, J. I. (1989). Teorías cognitivas del aprendizaje. Madrid: Ediciones Morata.

Putnam, L. (2001). Distance teamwork: The realities of collaborating with virtual colleagues. Online, 25(2), 54-58. Recuperado de: https://www.questia.com/magazine/1G170910886/distance-teamwork

Rojas, N; Carretero, M, \& Alvarez, I. (2012) Estrategia colaborativa de enseñanza de las matemáticas entre estudiantes de Ingeniería. uct [online]. 16(63), 085-092. Recuperado de: http://ve.scielo.org/scielo.php?pid=S1316-48212012000200002\&script=sci_abstract

Scardamalia, M., \& Bereiter, C. (1992). "Two models of classroom learning using a communal database". In S. Dijkstra, M. Krammer, \& J. Merriënboer, (Eds.). Instructional models in computer-based learning environments. Berlin: Springer-Verlag. Recuperado de: https://link.springer.com/chapter/10.1007/978-3-662-02840-7_14

Schellens, T., \& Valcke, M. (2006). Fostering knowledge construction in university students through asynchronous discussion groups. Computers \& Education, 46(4), 349-370. doi: 10.1016/j.compedu.2004.07.010

Sfard, A. (1988) On Two Metaphors for Learning and the Danger of Choosing Just One. Educational Researcher, 27(2), 4-13. doi: 10.2307/1176193

Thomas, R., \& MacGregor, K. (2005). Online project-based learning: How collaborative strategies and problem solving processes impact performance. Journal of interactive learning research, 16(1), 83-107. Recuperado de: https://www.researchgate.net/publication/277765128_Online_Project-

Based_Learning_How_Collaborative_Strategies_and_Problem_Solving_Processes_Impa ct_Performance 
Van Aalst, J. (2006). Rethinking the nature of online work in asynchronous learning networks. British Journal of Educational Technology, 37(2), 279-288. doi:10.1111/j.14678535.2006.00557.x

Van Boxtel, C., van der Linden, J., Kanselaar, G. (2000). Collaborative learning tasks and the elaboration of conceptual knowledge. Learning and Instruction, 10, 311- 330. doi: https://doi.org/10.1016/S0959-4752(00)00002-5

Van de Ven, A. \& Poole, M. (2002) Alternative Approach for Studying Organizational Change. Organization Studies, 26(9). doi: 10.1177/0170840605056907

Van Drie, J., van Boxtel, C., Jaspers, J., \& Kanselaar, G. (2005). Effects of representational guidance on domain specific reasoning in CSCL. Computers in Human Behavior, 21(4), 575-602. doi: https://doi.org/10.1016/j.chb.2004.10.024

Veerman, A. \& Veldhuis-Diermanse, E. (2001). Collaborative learning through computermediated communication in academic education. Recuperado de: http://eculturenet.org/mmi/euro-cscl/Papers/166.doc

Vicentini, I. (2020). La educación superior en tiempos de COVID-19: Aportes de la Segunda Reunión del Diálogo Virtual con Rectores de Universidades Líderes de América Latina. Banco Interamericano de Desarrollo. Washington DC. doi: http://dx.doi.org/10.18235/0002481

Zuluaga, J., Perez, F. \& Gomez, J. (2014). Matemáticas y TIC. Ambientes virtuales de aprendizaje en clase de Matemáticas. Recuperado de: recursos.portaleducoas.org/sites/default/files/VE14.014.pdf 